\title{
Synapsis in a paracentric inversion heterozygote of Chorthippus jacobsi (grasshopper)
}

\author{
M. DIEZZ \& J. L. SANTOS* \\ Departamento de Genética, Facultad de Biologia, Universidad Complutense, 28040 Madrid, Spain
}

\begin{abstract}
Analysis of surface-spread synaptonemal complexes of zygotene and pachytene spermatocytes was carried out on a grasshopper heterozygous for a paracentric inversion in chromosome L3. Inverted segments usually heterosynapse at pachytene without previous homosynapsis, although the possibility of synaptic adjustment in some nuclei is not excluded. A relationship between this synaptic behaviour and the absence of chromosome bridges and acentric fragments in the nine anaphase I cells analysed is established. The lack of reports concerning the existence of paracentric inversions in grasshopper natural populations is also discussed.
\end{abstract}

Keywords: heterosynapsis, meiosis, paracentric inversion, surface spreading.

\section{Introduction}

Surface spreading techniques have been extensively applied to a wide range of organisms to analyse the chromosome behaviour at early meiotic stages with special attention focused on the alterations produced in the normal pairing process by changes in chromosome number or structural rearrangements.

Using this procedure, Moses et al. (1982) described a peculiar synaptic process in paracentric inversion heterozygotes of mouse. It consists of the appearance of homologous synapsed loops at an early synaptic stage which are transformed into straight configurations through pachytene, even though a substantial proportion of the straightened loops must contain chiasmata. Therefore, the final synaptic configuration involves non-homologous synapsis of the inverted region.

The occurrence of this phenomenon, called 'synaptic adjustment', has also been reported in other paracentric and pericentric inversions (Davisson et al., 1981; Guichaoua et al., 1985; Tease \& Fisher, 1986; Borodin et al., 1990). However, this classical assumption seems to be inconsistent with several observations; for instance, homologous inversion loops remain the same throughout pachytene in a mouse heterozygous for a double inversion in chromosome 1 (Chandley, 1982), in a human male who carried a heterozygous

*Correspondence. pericentric inversion of chromosome 1 (Batanian \& Hulten, 1987), and in four different inversion heterozygotes of maize (Anderson et al., 1988). In other cases, the inverted chromosome region remains unpaired until late pachytene where heterosynapsis is achieved (Ashley et al., 1981) or non-homologous synapsis occurs even at early pachytene (Hale, 1986). It can be concluded from all these observations that inverted segments, at least in animals, do not follow a single pattern of synaptic behaviour at pachytene.

In this paper, we analyse, by means of a surface spreading technique, the chromosome pairing in a spontaneous paracentric inversion heterozygote of the grasshopper Chorthippus jacobsi. As far as we know this is the first study of this type carried out in insects.

\section{Materials and methods}

The paracentric inversion appeared in one individual among 25 last instar males examined of a wild population of Chorthippus jacobsi collected at the Campus of Complutense University, Madrid, Spain during June 1990.

Testes of these males were divided in two parts: one was fixed without previous treatment in acetic alcohol $1: 3$ and squash preparations were stained following a Giemsa C-banding technique described previously (Santos Giráldez, 1978), while another was subjected to a surface spreading method (J. L. Santos et al., unpublished observations). 


\section{Results}

Chorthippus jacobsi has 17 chromosomes $(16+\mathrm{X})$ in the male and $18(16+\mathrm{XX})$ in the female. Three pairs of autosomes (L1, L2 and L3) are long and submetacentric, whereas the remaining five pairs of autosomes and the X-chromosome are of medium to small size and are acrocentric.

The individual analysed here appeared among a sample of males used to study the chromosome pairing sequence in this species. The cytological analysis of 20 metaphase I and nine anaphase I cells by means of a $\mathrm{C}$-banding technique did not indicate any evidence of structural rearrangements. However, observations on surface-spread pachytene nuclei under the electron microscope revealed that the individual was heterozygous for a paracentric inversion located in the long arm of the L3 chromosome, near the centromere, because in two nuclei a characteristic loop indicative of homosynapsis in the inverted region was observed (Fig. $1 \mathrm{a}$ and $\mathrm{b}$ ). In another nucleus, a partially synapsed L3 bivalent that exhibited some non-homologous pairing within the inversion region was also seen (Fig. 2a and b). In the remaining 18 nuclei all bivalents showed straight pairing (Fig. 3) and thus non-homologous synapsis of the inverted segments was achieved.

Loop size was 14.5 and 8.3 per cent of L3 synaptonemal complex (SC) length in the nuclei in which autosomal SC lengths were 632 and $459 \mu \mathrm{m}$ respectively. As a progressive SC length shortening occurs

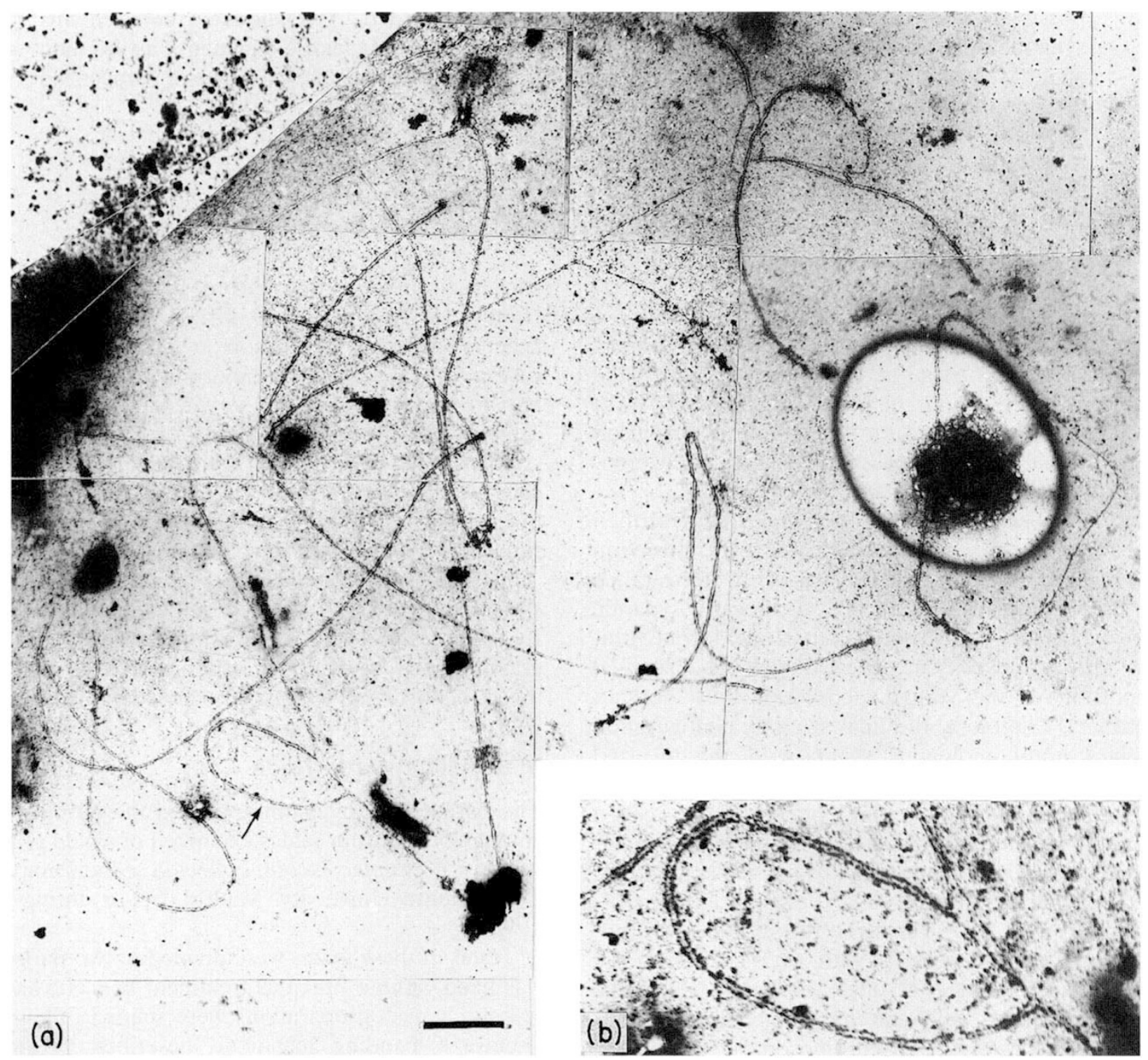

Fig. 1 (a) Electron micrograph of a silver-stained pachytene SC surface spread in which a loop indicative of homosynapsis in the inverted region is arrowed. Bar represents $5 \mu \mathrm{m}$. (b) Amplified detail of the loop. 


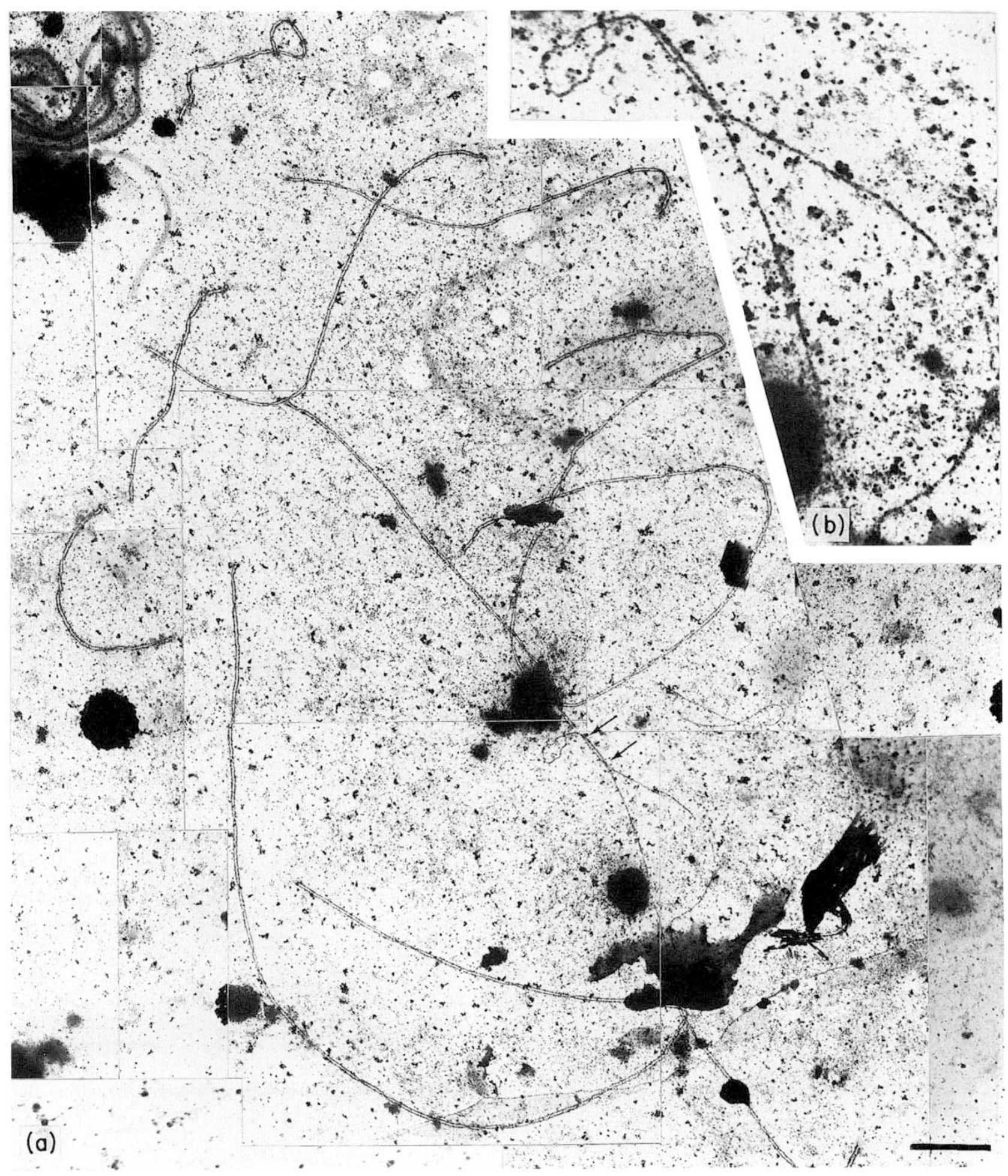

Fig. 2 (a) Electron micrograph of a silver-stained pachytene SC surface spread in which non-homologous pairing within the inversion region is observed (region between arrows). Bar represents $5 \mu \mathrm{m}$. (b) Amplified detail of inverted region showing asynapsis and non-homologous pairing.

throughout pachytene in this species (J. L. Santos et al., unpublished observations), loop size reduction could indicate the existence of a synaptic adjustment phenomenon. However, this observation must be con- sidered with caution due to the very low number of cells with loops analysed.

Inversion loops were absent in the eight zygotene nuclei in which all autosomal SCs could unequivocally 


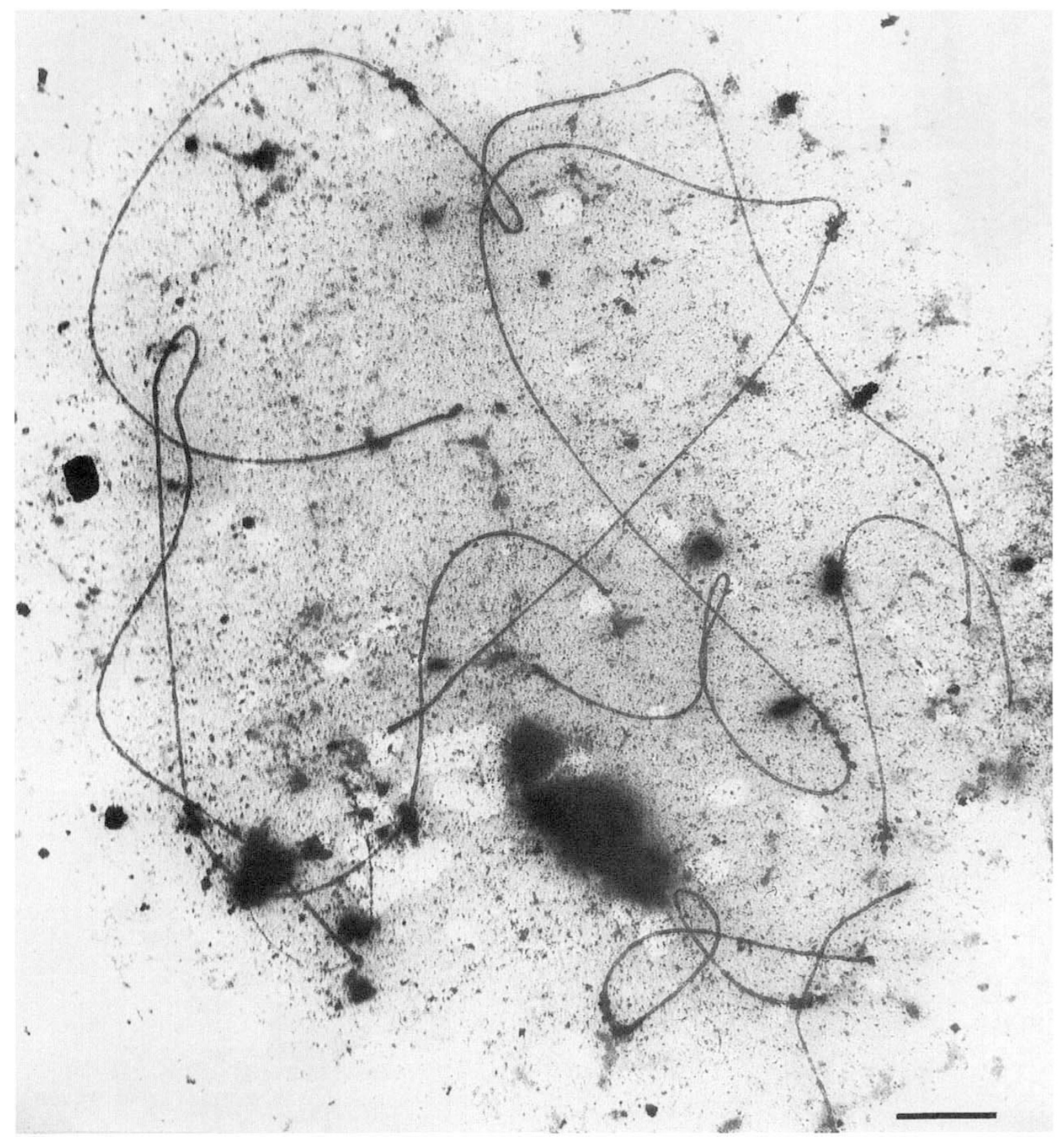

Fig. 3 Electron micrograph of a silver-stained pachytene SC surface spread in which all bivalents show straight pairing. Bar represents $7 \mu \mathrm{m}$.

be traced. Cells were considered to be at the zygotene stage when at least one bivalent other than L3 showed an unpaired region. Table 1 shows the percentage of chromosome pairing and the ratio between the length of the asynapsed region in the L3 bivalent and the total length of the bivalent in each nucleus. The most remarkable finding is that the L3 bivalent is extensively unpaired at zygotene when the other bivalents show much more complete pairing. These pairing difficulties may result in direct heterosynapsis although the possibility of late homosynapsis cannot be excluded.

\section{Discussion}

The L3 bivalent bearing the paracentric inversion can show two different configurations of pachytene: the 
Table 1 Zygotene date from the inversion heterozygote

\begin{tabular}{llll}
\hline $\begin{array}{l}\text { Nucleus } \\
\text { number }\end{array}$ & $\begin{array}{l}\text { Autosomal } \\
\text { haploid SC length } \\
(\mu \mathrm{m})\end{array}$ & Pairing $(\%)$ & A/L3* \\
\hline 1 & 757.5 & 75 & 45.7 \\
2 & 585 & 77 & 34 \\
3 & 626.2 & 83 & 41 \\
4 & 726 & 84 & 43 \\
5 & 527 & 86 & 32 \\
6 & 470 & 89 & 24 \\
7 & 406 & 91 & 17.5 \\
8 & 432.5 & 94.2 & 28 \\
\hline
\end{tabular}

${ }^{*} \mathrm{~A} / \mathrm{L} 3$ is the ratio between the length of the asynapsed region and that of the $\mathrm{L} 3$ bivalent.

presence of a loop in the long arm or straight pairing. Loops were the result of homologous synapsis between inverted segments (10 per cent of nuclei). The possibility that these loops were subject to synapsis adjustment could not be fully determined (Fig. 4). Straight pairing was observed in 90 per cent of nuclei, produced either by synaptic adjustment or by direct heterosynapsis. This latter possibility seems more feasible from the following considerations: (i) we have not observed loops at zygotene; (ii) if synaptic adjustment occurs we would expect a high frequency of loops at early pachytene and a progressive decrease in the number and size of the loops throughout this stage. However, we only found two loops in 21 pachytene nuclei, with SC lengths of $632-401 \mu \mathrm{m}$; (iii) the observation of an L3 pachytene bivalent showing a short non-homologous paired segment within the inversion (Fig. 2a and b) could be considered as the first step in the heterosynapsis process although this does not imply that heterosynapsis could also be achieved by pairing progression from the inversion-flanking regions. Nevertheless, the possibility of late homosynapsis of asynapsed zygotene regions, followed by synaptic adjustment, cannot be ruled out in some nuclei.

This synaptic behaviour may explain the lack of cytological evidence of an inversion in the metaphase I and anaphase I cells analysed under the following premises: (i) heterosynapsis prevents chiasma formation in the inversion loop and the subsequent appearance of chromosome bridges and acentric fragments; (ii) the size of the inversion is small enough to prevent chiasma formation, although as only nine anaphase I cells were observed it is difficult to say whether chiasmata occur in this region or not; and (iii) the inversion is located in the long arm near the centromere, a region that in normal individuals is usually devoid of chiasmata (J. L. Santos, unpublished results). Indeed, in 20 metaphase I
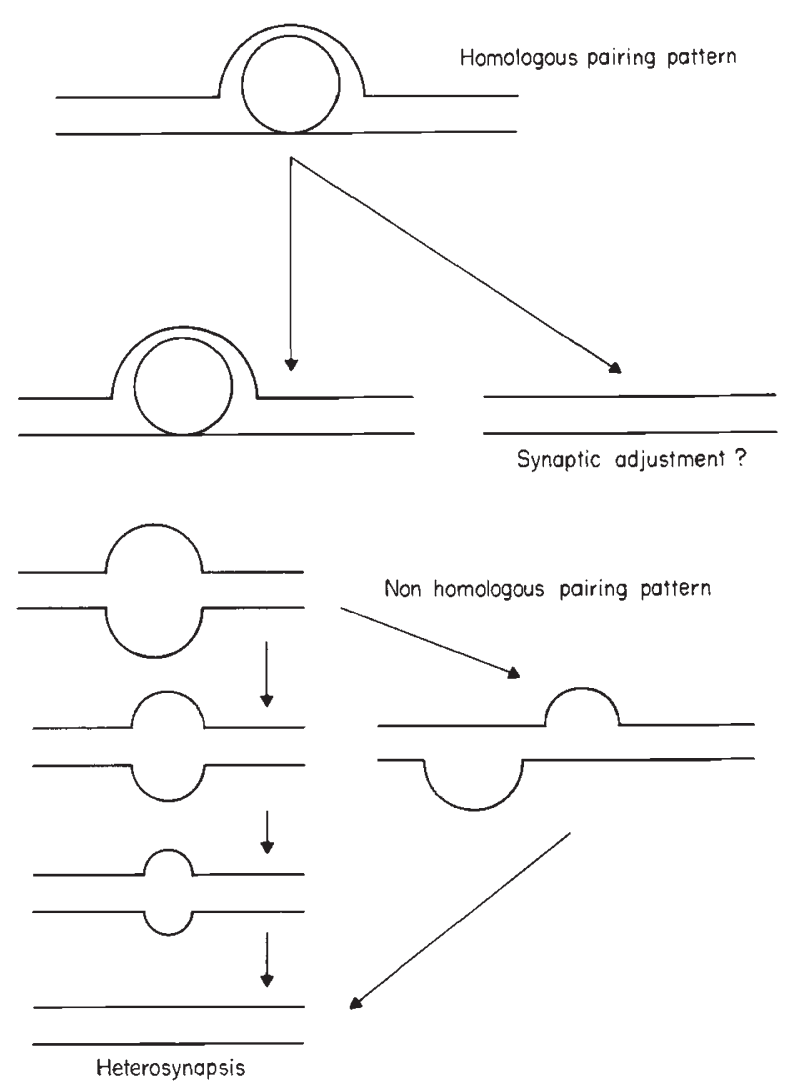

Fig. 4 A diagram showing the different pairing patterns displayed by the paracentric inversion.

L3 bivalents analysed no chiasmata were observed in this region.

The synaptic pattern of the Chorthippus jacobsi inversion displays a certain parallelism with those reported in other grasshopper species by means of classical light microscopical observations. Thus, Nur (1968) studied a male of Camnula pellucida that showed inversion loops in 4 per cent of the pachytenes and chromosome bridges and acentric fragments in approximately 8 per cent of the anaphase I cells examined, indicating that chiasmata do occur within the inversion. However, the most frequent pachytene configuration was the absence of a loop as a consequence of non-homologous pairing ( 88 per cent of cells) or asynapsis in the presumed inverted region ( 8 per cent of cells). A quantitative analysis in nine heterozygotes for a paracentric inversion in chromosome 2 of Boonacris alticola revealed 35 per cent of pachytene cells showing one asynaptic region, 5 per cent with reversed loops and the other 60 per cent showing straight pairing bivalents (Haines et al., 1978).

The major difference between these three cases is the existence of asynapsed regions in Camnula and Boonacris and their almost complete absence from 
Chorthippus jacobsi. It has been argued that these unpaired regions at pachytene could be due to the separation of homologues that had previously paired in a loop but in which no chiasmata had formed (Martin, 1967). Taking into account the synaptic behaviour displayed by different inversions (see Anderson et al., 1988 for references), it is perhaps more reasonable to assume that in Camnula and Boonacris those cells showing unpaired regions are at early pachytene and they will progress towards a heterosynaptic stage by the end of this phase. Observations in Boonacris, where asynaptic regions showed variable length from 10 to 50 per cent of the length of the bivalent, would be in agreement with this hypothesis. On the other hand, it is also possible that these unpaired regions will remain the same from early to late pachytene as occurs in maize inversions (Anderson et al., 1988).

Note that whereas in grasshopper populations pericentric inversions are seemingly abundant (Hewitt, 1979), it is generally accepted that paracentric inversions are very scarce (White, 1973). Indeed, only the three cases discussed here are known to date. As has been pointed out by Haines et al. (1978), the question is not the absence of paracentric inversions in grasshoppers but the difficulty of detecting them as a consequence of their peculiar meiotic behaviour. Undoubtedly, surface spreading techniques provide a powerful method for detecting and analysing these types of rearrangements, especially those of small size.

\section{Acknowledgements}

This paper was supported by a grant PB 88-0121 awarded by the Comision Interministerial de Ciencia y Tecnologia (Spain). Dr Nieves Cuñado, José Barrios, Angel del Cerro, Ana Fernández, Agustin Fernández and the staff of the Complutense University Electron Microscope Unit, especially Dr Carlos Barba, provided valuable assistance.

\section{References}

ANDERSON, L. K., STACK, S. M. AND SHERMAN, J. M. 1988. Spreading synaptonemal complexes from Zea mays. I. No synaptic adjustment of inversion loops during pachytene. Chromosoma, 96, 295-305.
ASHLEY, T., MOSES, M. J. AND SOLARI, A. J. 1981. Fine structure and behaviour of a pericentric inversion in the sand rat Psammomys obesus. J. Cell Sci., 50, 105-119.

BATANIAN, J. AND HULTEN, M. A. 1987. Electron microscopic investigations of synaptonemal complexes in an infertile human male carrier of a pericentric inversion $\operatorname{Inv}(1)$ (p32q42). Hum. Genet., 76, 81-89.

BORODIN, P. M., GORLOV, I. P. AND LADYGINA, T. Y. 1990. Synapsis in single and double heterozygotes for partially overlapping inversions in chromosome 1 of the house mouse. Chromosoma, 99, 365-370.

CHANDLEY, A. C. 1982 . A pachytene analysis of two malefertile paracentric inversions in chromosome 1 of the mouse and in the male-sterile double heterozygote. Chromosoma, 85, 127-135.

DAVISSON, M. T., POORMAN, P. A., RODERICK, T. H. AND MOSES, M. J. 1981. A pericentric inversion in the mouse. Cytogenet. Cell Genet., 30, 70-76.

GUichaoua, M. R., DElafontaine, D., TAURelle, R., TAILlemite, J. L., MORAZZANI, M. R. AND LUCIANI, J. M. 1985. Loop formation and synaptic adjustment in a human male heterozygous for two pericentric inversions. Chromosoma, 93, 313320.

haines, R. L., Roberts, P. A. AND LAtTin, J. D. 1978. Paracentric inversion polymorphism in the grasshopper Boonacris alticola. Chromosoma, 65, 185-197.

HALE, D. W. 1986. Heterosynapsis and suppression of chiasmata within heterozygous pericentric inversions of the Sitka deer mouse. Chromosoma, 94, 425-432.

HEwIT,, G. M. 1979. Animal Cytogenetics, 3. Insecta, 1. Orthoptera. Gebrüder Borntraeger, Berlin.

MARTIN, J. 1967. Meiosis in inversion heterozygotes in Chironomidae. Can. J. Genet. Cytol., 9, 255-268.

MOSES, M. J., POORMAN, P. A., RODERICK, T. H. AND DAVISSON, M. T. 1982. Synaptonemal complex analysis of mouse chromosomal rearrangements iv. Synapsis and synaptic adjustment in two paracentric inversions. Chromosoma, 84, 457-474.

NUR, U. 1968. Synapsis and crossing over within a paracentric inversion in the grasshopper Camnulla pellucida. Chromosoma, 25, 193-214.

SANTOS, J. L. AND GIRALDEZ, R. 1978. The effect of C-heterochromatin in chiasma teminalisation in Chorthippus biguttulus L. (Acrididae, Orthoptera). Chromosoma, 70, 59-66.

TEASE, C. AND FISHER, G. 1986. Further examination of the production-line hypothesis in mouse foetal oocytes. Inversion heterozygotes. Chromosoma, 93, 447-452.

white, M. J. D. 1973. Animal Cytology and Evolution, 3rd edn. Cambridge University Press, Cambridge. 